\title{
Analysis of water absorbency into knitted spacer structures
}

Tilak Dias and G B Delkumburewatte

\author{
William Lee Innovation Centre, Textile and Paper, School of Materials, The University \\ of Manchester, Manchester, UK, tilak.dias@manchester.ac.uk, \\ gune.del@postgrad.manchester.ac.uk
}

\begin{abstract}
The absorbency properties of knitted structures are very important in designing garments that both remove liquid sweat from the skin and provide tactile and sensorial comfort to the wearer. Water absorbency by knitted spacer structures was experimentally investigated using a gravimetric absorbency tester to record absorbency rate, total absorbency, and time taken to saturate the structure. The geometry of spacer structures was analyzed and a model created to define the capillary characteristic in the spacer yarn. Absorbency into the spacer structures was modeled using the fabric parameters, the capillary radius, and the properties of water. Experimental and theoretical results were compared to validate the models.
\end{abstract}

\section{Key words}

Absorbency, knitted spacer fabrics, moisture management, model, capillary, total absorbency, absorbency rate

\section{Introduction}

Textile structures play a very important role as liquid accumulators and as a medium for liquid transport in technical and medical applications as well as in the field of clothing. Normally the removal of sweat also helps to reduce heat stress together with sweat evaporation which provides a major source of cooling to the body. Evaporative cooling is not possible for entirely enclosed protective clothing such as CBRN (Chemical, Biological, Radiological or Nuclear) or firefighter clothing, and so it is important to remove sweat from the skin to maintain tactile and sensorial comfort while wearing the garment. The study of absorption has become more important as textile structures are 
frequently used for medical and technical applications, particularly with the introduction of new technologies and developments.

Experimental work on liquid absorbency with different structures has been carried out in the last fifty years to establish the relationship between the absorbency rate of structures the absorbency property of fibres, the structure of the fabric, the wetting of fibres and the properties of the liquids involved. However, it seems that further theoretical work needs to be carried out to engineer structures for better moisture management.

One of the objectives of this paper is to present the experimental results of an absorbency test based on the gravimetric absorbency principle for special spacer structures that we have constructed for moisture management under extreme conditions. A model was created to describe the capillaries in a spacer yarn, and a second model was created to describe the absorbency within a spacer structure. In creating the models the fabric specification such as courses and wales per unit length, stitch length, fabric thickness, yarn diameter, and liquid properties were considered.

\subsection{Literature review}

Absorbency is the ability of a textile structure to absorb water. In textile structures, water is absorbed due to two phenomena, namely wetting and diffusion. Liquid absorption into a porous textile structure depends on the following;

- Properties of the liquid such as viscosity, density and surface tension

- Fibre surface wetting characteristics due to their hydrophobic or hydrophilic nature. Capillary forces are generated as a result of wetting. Spontaneous wetting of the fibre surface by a liquid is known as wicking [1]

- The geometric parameters of the fibre assembly, such as thickness, porosity, and pore size or capillary radii.

The surface tension of the liquid causes a pressure difference $(\Delta P)$ across the liquid surface in the capillary known as the capillary force, which is given by the Laplace equation [2], [3]:

$$
\Delta P=\frac{2 \gamma \cos \theta}{r}
$$


where, $r$ = radius of capillary; $\gamma=$ surfacetension of the liquid; $\theta=$ contact angle.

As the liquid in the capillary rises, an opposing pressure head $\left(\Delta P_{h}\right)$ is created due to the weight of the risen liquid, which depends on the density of the liquid $(\delta)$, acceleration due to gravity (g), and the height of liquid rise (L).

$$
\Delta P_{h}=L g \delta
$$

Therefore, the actual pressure gradient change $(\Delta P)$ with the rising water level is:

$$
\Delta P=\frac{2 \gamma \cos \theta}{r}-L g \delta
$$

Liquid flow into a porous structure is dependent on contact angle, physical capillary radius, and density and viscosity of the liquid. The volumetric liquid flow through a textile structure can be determined by employing Hagen-Poiseuille's law for laminar flow [4]:

$$
\frac{d V}{d t}=\frac{\pi r^{4}}{8 \eta L} \Delta P
$$

where,

$$
\begin{aligned}
& \Delta P=\text { net pressure gradient; } \\
& L=\text { length of liquid rise; } \\
& \eta=\text { vis cos ity of liquid. }
\end{aligned}
$$

Combining equations (3) and (4):

$$
\frac{d V}{d t}=\left(\frac{2 \gamma \cos \theta}{r}-L g \delta\right) \frac{\pi r^{4}}{8 \eta L}
$$

Lucas and Washburn developed an equation based on Hagen-Poiseuille's equation considering $\mathrm{dV}=\mathrm{dL} \cdot \pi \cdot \mathrm{r}^{2}$ for linear flow rate $(d L / d t)$ in equilibrium [5]:

$$
\frac{d L}{d t}=\frac{r \gamma \cos \theta}{4 \eta L}
$$

Taking the influence of gravity due to the risen liquid, the flow rate becomes:

$$
\frac{d L}{d t}=\frac{r \gamma \cos \theta}{4 \eta L}-\frac{r^{2} g \delta}{8 \eta}
$$


After integration the Lucas-Washburn equation (6) can be written as:

$$
L=\sqrt{\frac{r \gamma \cos \theta}{2 \eta}} \sqrt{t}
$$

This equation can be simplified to

$$
L=K \sqrt{t}
$$

where, $\quad K=$ Rate Const an $t=\sqrt{\frac{r \gamma \cos \theta}{2 \eta}}$

Most of the experimental work carried out by previous researchers, shows that the penetration of liquid into a cylindrical capillary is proportional to the square root of time, the relationship is known as Lucas-Washburn kinetics. However, Laughlin [6] suggested that the time exponent, $\mathrm{k}$, is less than 0.5 , and modified the equation to:

$$
\mathrm{L}=\mathrm{C} \mathrm{t}^{\mathrm{k}}
$$

According to equation (8), the height of the water will rise continuously with time, but in practice the water column rises until the surface tension is equal to the weight of the water column.

$$
\ln (\mathrm{L})=\ln (\mathrm{C})+\mathrm{K} \ln (\mathrm{t})
$$

Laughlin plotted the equation and obtained a straight line.

The change of contact angle with increase of water level [7, 8], the moment of inertia [9], and the effect of gravity [10], have all been ignored and provide the basis of the controversy over the Washburn equation. After experiments with various surfactant solutions, Hodgson and Berg [11] found that liquids obey the Washburn theory despite the limitations mentioned above. Consequently, the Lucas-Washburn wicking kinetics relationship is still widely accepted.

Several researchers $[12,13,14]$ tried to accommodate the effect of gravity into the Lucas Washburn theory. In 1988 the theory developed by Landau [15] is a special form of Hagen Poiseuille's law for laminar viscous flow. This theory gives the rate of liquid rise, considering gravity and the angle of capillary to the vertical as: 


$$
\frac{\mathrm{dh}}{\mathrm{dt}}=\frac{\mathrm{r} \gamma \cos \theta}{4 \mu \mathrm{h}}-\frac{\mathrm{r}^{2} \zeta \mathrm{g} \cos \beta}{8 \mu}
$$

where, $\zeta$ is liquid density, $\mu$ is the viscosity of the liquid, $\beta$ is the angle of the capillary to the vertical tube axis and $h$ is the distance travelled by the liquid measured from the reservoir along the tube axis.

Landau decided that equation (13), as a non-linear ordinary differential equation, can be solved only by ignoring the parameter $\frac{\mathrm{r}^{2} \zeta \mathrm{g} \cos \beta}{8 \mu}$. This will be correct for horizontal liquid penetration or for smaller values of $r$, when the equation after integration will give the distance travelled by the liquid as a function of time as, $h=\sqrt{\frac{r \gamma \cos \theta}{2 \mu}} t$, and this equation is the same as the Lucas Washburn equation (8).

In 1988, Joos et al [8] investigated experimentally the changes of the contact angle and proved that the contact angle is dynamic and becomes lower as the liquid rises. They proved that the dynamic contact angle $\left(\cos \theta_{d}\right)$ changes with the surface tension $(\sigma)$, viscosity $(\eta)$ and the velocity of the meniscus $(v)$ as below:

$$
\cos \theta_{\mathrm{d}}=\cos \theta_{0}-2\left(1+\cos \theta_{0}\right)\left(\frac{\eta \mathrm{v}}{\sigma}\right)^{1 / 2}
$$

where $\theta_{0}$ is the static advancing contact angle.

In 1997, Abraham et al [13], studied the kinetics of vertical liquid penetration into a capillary which initially contains a fluid of negligible viscosity (usually gas) by considering gravity, and gave the equation for the liquid rise $(h)$ with time $(t)$ as

$$
\mathrm{At}=-\mathrm{Bh}-\ln (1-\mathrm{Bh})
$$


where $A=\frac{\rho^{2} g^{2} r^{3}}{16 \sigma \mu \cos \theta}, B=\frac{\rho g r}{2 \sigma \cos \theta}$, and $\rho$ is the density of the liquid, $\sigma$ is the surface tension, $\mu$ is the viscosity, $\cos \theta$ is the contact angle, $r$ is the radius of capillary and $\mathrm{g}$ is gravity.

In 2006 Nyoni and Brook [14] studied the wicking mechanism in vertical yarns using textured twisted and untwisted filament yarns under variable tension. They concluded that the wicking performance or the wicking height increases with the increase of tension and decreases with the increase of twist. Further, they concluded that the penetration and retention of liquid is affected by the heterogeneity of pore size, shape and orientation. After microscopic examination, they concluded that there are unsaturated, saturated and dry zones in the yarns.

The two dimensional Ising's model and the Monte Carlo simulation were used by Zhong et al [15] to describe the wetting process. They noted that the travelling rate of liquid is higher in the area where the packing density is higher, and the width of the liquid column decreases with height due to the balance of surface tension and gravity.

In 2006, Umair Saeed [16] studied the liquid uptake by various knitted structures, such as plain and spacer, with different yarns. The theoretical model for liquid uptake considering gravity was further developed and the liquid absorbency rate given as a function of time by:

$m=\frac{\rho_{l}}{\rho_{y}}\left(\frac{\phi}{1-\phi}\right) A x\left[1-\exp \left(-\frac{2 \gamma \cos \left(\theta_{0}\right) r}{8 \eta L} t\right)\right]$

where $\mathrm{A}$ is the fabric area, $\mathrm{x}$ is the fabric weight, $\phi$ is the porosity of the fabric, $\rho_{1}$ is the density of the liquid, and $\rho_{y}$ is the density of the yarn.

Figure 1 shows an example for the theoretical and experimental liquid uptake of a knitted fabric sample [16]. 


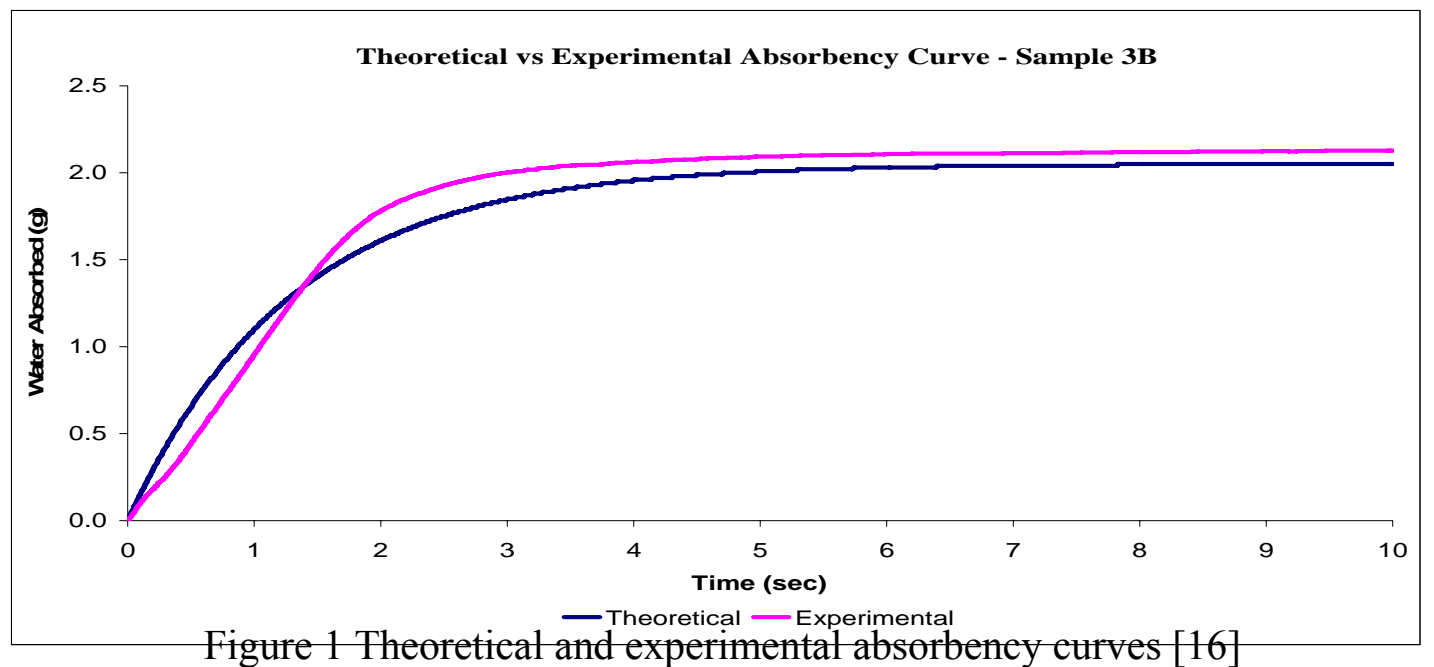

Figure 1 Theoretieal and experimental absorbeney eurves [16]

\section{Methods and Apparatus}

Geometrical analysis explains the theoretical capillary radii of spacer structures. Liquid take-up rate is calculated considering capillary radii and the gravitational force. In the experimental part, the liquid take-up of different spacer structures was determined using the Allasso Absorbency Analyzer shown in Figure 2, based on the gravimetric absorbency testing system. Different types of spacer structure were knitted with a different number of floats and a different number of spacer yarns in between two single jersey courses in front bed and back bed on the 7 gauge Shima Seiki 122-S flat bed knitting machine.

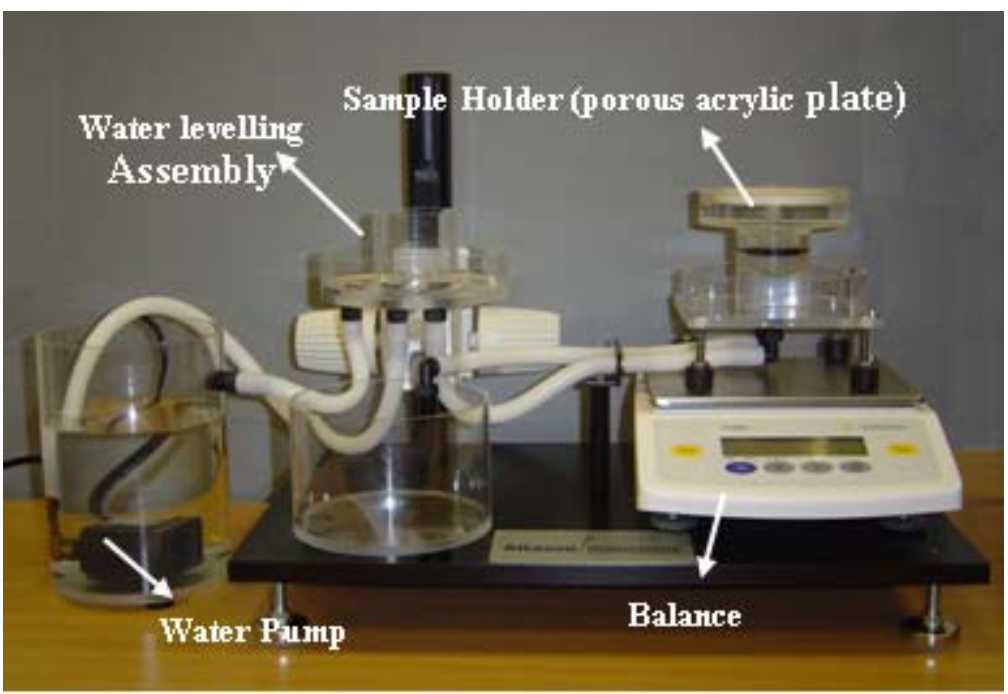

Figure 2 The Allasso Absorbency Analyzer 


\subsection{Theoretical modelling of a spacer structure for capillary radius and the rate of liquid absorbency.}

The capillary radius model has been created with reference to the geometry of the spacer structure. Fabric thickness, fabric structure, course spacing, wale spacing, yarn crimp, the number of filaments in the yarn, and the yarn counts are the parameters considered in modelling. The second part of the model was concerned with the percentage of liquid uptake with time, applying the Washburn equation on the basis of capillary radius, fabric thickness, and the angle of the capillaries in the structure.

\subsubsection{Capillary radius}

For the calculation of capillary radius, it was assumed that the spacer yarn in the spacer fabric lies in a zigzag path between front bed and back bed as shown in the schematic diagram. It is also assumed that the filaments are equally distributed and parallel to the yarn direction, as the spacer yarn is not twisted in the spacer structure.

The shapes of spaces formed by filaments in a cross-section of tightly packed bundle of parallel fibres are approximately smaller triangles or unstable squares. However, the motions of viscous fluid through linear channels do not depend critically on the shape when the ratio of volume and the exposed area is considered [17]. Therefore, in this work it is assumed that spaces are circular and are equivalent to average area of spaces in the bundle. Consequently, we can assume that the air space in the spacer structure consists of very fine circular capillaries, which are parallel to the spacer yarn and have the same length as the spacer yarn.

We will consider a cuboid sector of fabric with the surface having a rectangle $1 \mathrm{~cm}$ long and the width containing a number of wales, together with one spacer yarn running the full length from front bed to back bed. Generally the spacer yarn produces tuck stitches forming a zigzag layout.

If the number of needle trick displaced from one tuck in the front bed to the next tuck in the back bed (floats) is $\mathrm{S}$, then the yarn makes an $\mathrm{S} \times \mathrm{S}$ zigzag layout as shown in Figure 3. 
It is assumed that $\mathrm{b}$ is the fabric thickness and $\mathrm{z}$ the distance between two wales, where two adjacent tucks are formed from a yarn, one is in the front bed and the other in the back bed.

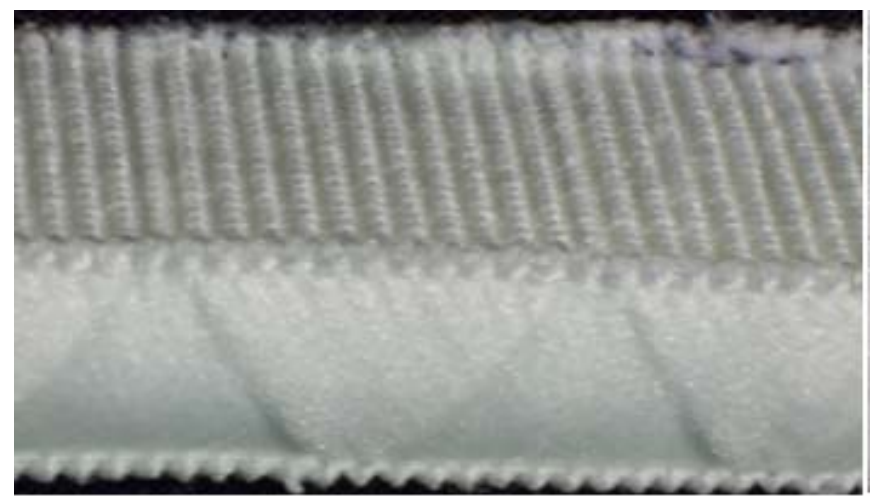

a) Photo of a spacer fabric

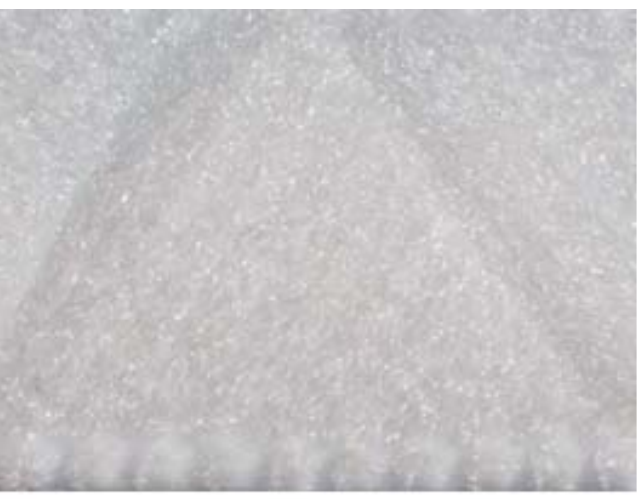

b) Enlarged photo of the cross-

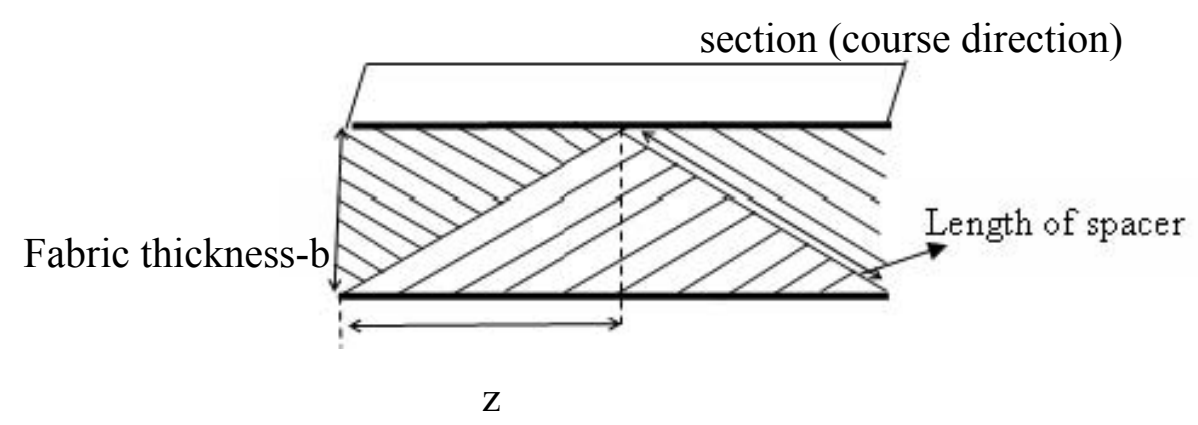

c) Diagram of spacer yarns packing in the fabric in course direction

Figure 3 Course direction cross- section of knitted spacer fabric

The width $\mathrm{z}$ is given by;

$$
\mathrm{z}=\mathrm{S} * \text { width of wale }(\mathrm{W})=\mathrm{S} / \mathrm{w}
$$

where $\mathrm{w}$ is wales per $\mathrm{cm}$.

The assumptions made in this capillary model are as below:

1. The spacer yarn within the structure from front bed to back bed lay straight.

2. The filaments within the textured yarn are not straight and are crimped.

3. Filaments are equally distributed within the yarns as there are no external compressing forces. 
4. Spaces between filaments within a yarn and spaces between filaments of adjacent yarns are identical because of the extremely close packing of the whole spacer structure (Figure 3.b). Observation made using Projectina microscope shows that the spaces between inter yarn and intra yarn filaments are similar when the spacer yarns are tightly packed.

The volume of the fabric sample can be considered as V;

$$
\mathrm{V}=\text { thickness } * \text { width } * \text { length of fabric }=\text { b. } \mathrm{z} .1=\mathrm{b} .1 . \mathrm{S} / \mathrm{w} \mathrm{c \textrm {cm } ^ { 3 }}
$$

The averages of wet and dry fabric thicknesses are calculated after measuring under a pressure of $1 \mathrm{kPa}$. Fabric thickness is measured using fabric samples placed between two plates under a pressure of $500 \mathrm{~g} / 50 \mathrm{~cm}^{2}(1 \mathrm{kPa})$. The variations between dry and wet fabrics are very small when samples are properly relaxed.

Then the length of yarn between two tucks is

$$
\mathrm{L}=\sqrt{ }\left(\mathrm{b}^{2}+\mathrm{z}^{2}\right)=\sqrt{ }\left[\mathrm{b}^{2}+(\mathrm{S} / \mathrm{w})^{2}\right]
$$

If we consider the fibre volume $\left(\mathrm{V}_{\mathrm{m}}\right)$ and the fibre weight $\left(\mathrm{m}_{\mathrm{m}}\right)$

Volume of material $\left(\mathrm{V}_{\mathrm{m}}\right)=$ weight of yarn / fibre density

Weight of material $\left(\mathrm{m}_{\mathrm{m}}\right)=$ Total yarn length $*$ yarn count

Thus, $\mathrm{V}_{\mathrm{m}}=\left[\right.$ rows $\left.* \mathrm{c} * \sqrt{ }\left(\mathrm{b}^{2}+\mathrm{z}^{2}\right)\right] / 10^{5} *$ Tex $/ \rho_{\mathrm{f}}$

where (rows) is the number of spacer courses between two single jersey courses in the front or back bed.

Air space within the considered unit $=$ Total volume- volume of the material

Air space $=\mathrm{b} * 1 / \mathrm{w} * \mathrm{~S}-\left[\right.$ rows $\left.* \mathrm{c} * \sqrt{ }\left(\mathrm{b}^{2}+\mathrm{z}^{2}\right)\right] / 10^{5} *$ Tex $/ \rho_{\mathrm{f}}$

Generally the number of capillaries in a yarn is equal to the number of filaments in the yarn less the number of filaments on the surface of the yarn if cylindrical packing occurs. However, in the case of closely packed spacer structures, filaments on the surface form capillaries with the surface filaments from adjacent yarns. Therefore the total number of capillaries is equal to the total number of filaments in the yarn and then the total volume of capillaries is equal to the air space in the fabric occupied by the yarn. 
The air space $=$ Total number of capillaries $*$ length $*$ area of one capillary

Then the total number of individual filaments in the yarn is 170 as five fold $1 / 167 / 34$ polyester yarn was used as the spacer fibre. The total number of capillaries within the yarn is also considered to be 170 , and hence the total number of capillaries within the unit is equal to $170 *$ rows $* \mathrm{c}$.

Therefore the volume of air space $=(170 *$ rows $* c) * \sqrt{ }\left(b^{2}+z^{2}\right) * \pi r^{2}$

Comparing the two equations (21) and (22)

$(170 *$ rows $* c) * \sqrt{ }\left(b^{2}+z^{2}\right) * \pi r^{2}=b * 1 * S / w-\left[\right.$ rows $\left.* c * \sqrt{ }\left(b^{2}+z^{2}\right)\right] / 10^{5} *$ Tex $/ \rho_{\mathrm{f}}$

$\mathrm{r}^{2}=\mathrm{b} * 1 / \mathrm{w} * \mathrm{~S}-\left[\right.$ rows $\left.* \mathrm{c} * \sqrt{ }\left(\mathrm{b}^{2}+\mathrm{z}^{2}\right)\right] / 10^{5} *$ Tex $/ \rho_{\mathrm{f}}$

$170 *$ rows $* \mathrm{c} * \sqrt{\left(\mathrm{b}^{2}+\mathrm{z}^{2}\right) * \pi}$

$r=\sqrt{\frac{b \cdot \frac{1}{w} \cdot S-\left[\text { rows.c. } \sqrt{\left(b^{2}+z^{2}\right)}\right] / 10^{5} \cdot T / \rho_{f}}{170 . \text { rows.c. } \sqrt{\left(b^{2}+z^{2}\right)} \cdot \pi}}$

$r=\sqrt{\frac{b \cdot \frac{1}{w} \cdot S-\left[\text { rows.c. } \sqrt{\left.\left(b^{2}+\frac{S^{2}}{w^{2}}\right)\right] / 10^{5} \cdot T / \rho_{f}}\right.}{170 . \text { rows.c. } \sqrt{\left(b^{2}+\frac{S^{2}}{w^{2}}\right) \cdot \pi}}}$

In the above calculation we have not considered the crimp of the filament. In our case the spacer yarns are textured and have high crimp, and also the yarns are in a relaxed state. Therefore, we have to consider the crimp to the material length, and the formula for capillary radius becomes:

$r=\sqrt{\frac{b \cdot \frac{1}{w} \cdot S-\left[\text { rows.c. } \sqrt{\left(b^{2}+\frac{S^{2}}{w^{2}}\right) \cdot 1 /(1-\text { crimp })}\right] / 10^{5} \cdot T / \rho_{f}}{170 . \text { rows.c. } \sqrt{\left(b^{2}+\frac{S^{2}}{w^{2}}\right) \cdot \pi}}}$, 


\subsubsection{Liquid absorption into spacer structures}

The theoretical model is created to define the liquid take-up into spacer structures considering the capillaries formed by the filament spacer yarn as explained previously. Hagen-Poiseulle's law based on the Washburn equation for upward liquid take-up by the structure was applied in creating a model for liquid uptake considering the radius of capillaries within the structure.

$$
d V=\frac{\pi \sum P}{8 \eta \ell}(r)^{4} . d t
$$

where $\mathrm{V}$ is volume, $\Sigma \mathrm{p}$ is pressure difference, $\mathrm{r}$ is capillary radius, $\eta$ is viscosity of the liquid and $\ell$ is liquid rise.

$d V=\pi r^{2} d \ell$

$\pi r^{2} d \ell=\frac{\pi \sum P}{8 \eta \ell}(r)^{4} \cdot d t$

$d \ell=\frac{\sum P}{8 \eta \ell}\left(r^{2}\right) d t$

$\sum P=$ Capillary pressure - hydraulic pressure

We assume that the fabric thickness is $\mathrm{b}$, and that $\mathrm{z}$ is the distance between two wales, where two adjacent tucks are formed from a yarn. The angle between fabric surface and the direction of the tuck yarn is $\varphi$ as shown in Figure 4.

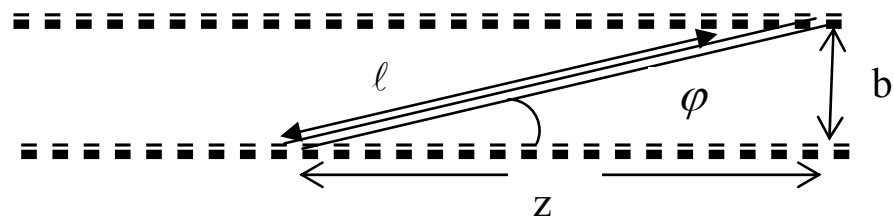

Figure 4 Schematic diagram of a capillary formed by spacer yarn in the structure

Then $\sin \varphi=\frac{b}{\sqrt{b^{2}+z^{2}}}$

If the liquid rise along the capillary is $\ell$ then the hydraulic pressure can be given as; 
$\lg \rho_{w} \sin \varphi=\lg \rho_{w} \frac{b}{\sqrt{b^{2}+z^{2}}}$ where $\rho_{w}$ is the density of water and $g$ is gravity.

Then the total pressure difference is,

$\sum P=\frac{2 \gamma}{r} \cos \theta-\lg \rho_{w} \sin \varphi$

$\sum P=\frac{2 \gamma}{r} \cos \theta-\lg \rho_{w} \frac{b}{\sqrt{b^{2}+z^{2}}}$

Hagen-Poiseuille's law can then be applied to the liquid rise in the capillaries in the spacer structure as:

$d \ell=\frac{\frac{2 \gamma}{r} \cos \theta-\lg \rho_{w} \frac{b}{\sqrt{b^{2}+z^{2}}}}{8 \eta \ell}\left(r^{2}\right) d t$

$\frac{\ell d \ell}{\frac{2 \gamma}{r} \cos \theta-\lg \rho_{w} \frac{b}{\sqrt{b^{2}+z^{2}}}}=\frac{\left(r^{2}\right)}{8 \eta} d t$

$\int \frac{\ell}{\frac{2 \gamma}{r} \cos \theta-\lg \rho_{w} \frac{b}{\sqrt{b^{2}+z^{2}}}} d \ell=\int \frac{\left(r^{2}\right)}{8 \eta} d t$

For simplifying purposes, we assume, $A=\frac{2 \gamma}{r} \cos \theta, B=g \rho_{w} \frac{b}{\sqrt{b^{2}+z^{2}}}$ and $K=\frac{\left(r^{2}\right)}{8 \eta}$ Then,

$$
\begin{aligned}
& \int \frac{\ell}{A-\ell B} d \ell=\int K d t \\
& \frac{-1}{B}\left[\ell+\frac{A}{B} \ln (A-B \ell)\right]+C=K . t \\
& t=0 \rightarrow \ell=0, \quad t=\infty \rightarrow \ell=\sqrt{b^{2}+z^{2}}, \\
& C=\frac{A}{B^{2}} \ln (A)
\end{aligned}
$$


$B \cdot \ell+A \cdot \ln \left[1-\frac{B \cdot \ell}{A}\right]=-K \cdot B^{2} t$

$g \cdot \rho_{w} \cdot \frac{b}{\sqrt{b^{2}+z^{2}}} \cdot \ell+\frac{2 \gamma}{r} \cdot \cos \theta \cdot \ln \left[1-\frac{g \cdot \rho_{w} \cdot r}{2 \gamma \cdot \cos \theta} \cdot \frac{b}{\sqrt{b^{2}+z^{2}}} \ell\right]=-\frac{r^{2}}{8 \eta} \cdot\left[g \cdot \rho_{w} \cdot \frac{b}{\sqrt{b^{2}+z^{2}}}\right]^{2} \cdot t$.

Equations (42) and (43) are not linear equations and they can be considered as Lambertw functions or Omega functions. These types of equations can be solved using MatLab or Mathematica software. Equations (44) and (45) are solutions given by MatLab software, for equations (42) and (43) respectively.

$$
\begin{gathered}
\ell(\mathrm{t})=\text { A.lambertw }\left(-\exp \left(-\left(\mathrm{K} \cdot \mathrm{B}^{2} \cdot \mathrm{t}+\mathrm{A}\right) / \mathrm{A}\right)+1\right) / \mathrm{B} \ldots \ldots \ldots \ldots \ldots(44) \\
\ell(\mathrm{t})=\frac{2 \gamma}{\mathrm{r}} \cos \theta \cdot \operatorname{lambertw}\left[-\exp \left(-\left(\frac{\mathrm{r}^{2}}{8 \eta} \cdot\left(\mathrm{g} \cdot \rho_{\mathrm{w}} \frac{\mathrm{b}}{\sqrt{\mathrm{b}^{2}+\mathrm{z}^{2}}}\right)^{2} \cdot \mathrm{t}+\frac{2 \gamma}{\mathrm{r}} \cos \theta\right) / \frac{2 \gamma}{\mathrm{r}} \cos \theta\right)+1\right] \\
\quad / \mathrm{g} \rho_{\mathrm{w}} \frac{\mathrm{b}}{\sqrt{\mathrm{b}^{2}+\mathrm{z}^{2}}}
\end{gathered}
$$

\subsubsection{Theoretical liquid absorbency rate into spacer structures}

After finding the length of capillary flow with time, the mass of the water flow through the capillary can be obtained by multiplying with the area of capillary cross-section and the density of water, thus:

$$
m_{w}=\pi \cdot r^{2} \cdot \rho_{w} \cdot \ell(t)
$$

If we consider the total number of capillaries in one spacer yarn, we simply multiply by the total number of filaments. So the total water flow in a spacer yarn is,

$$
m_{w}=170 \cdot \pi \cdot r^{2} \cdot \rho_{w} \cdot \ell(t)
$$

The percentage liquid absorbency rate can be calculated considering the liquid absorbency rate and the weight of the spacer yarn.

Liquid take up percentage $=\mathrm{m}_{\mathrm{w}} / \mathrm{m}_{\mathrm{m}} * 100$ 
Therefore we have to calculate the weight of the spacer yarn and the weight of the water absorbed along the yarn from one surface to the other surface. After considering the crimp of the spacer yarn, the length of spacer yarn from the front bed needle to the back bed needle can be given as:

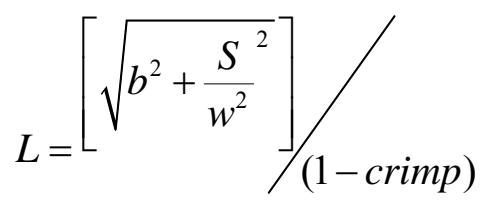

The weight of the spacer yarn = yarn length* yarn count

The weight of yarn $m_{m}=\frac{\operatorname{Tex} \cdot 10^{-5} \cdot \sqrt{b^{2}+\frac{S^{2}}{w^{2}}}}{(1-\operatorname{crimp})}$

The liquid take up rate $=\frac{170 \cdot \pi \cdot r^{2} \cdot \rho_{w} \cdot \ell(t) \cdot(1-\text { crimp })}{\sqrt{b^{2}+\frac{\text { spaces }^{2}}{w^{2}}} \cdot 100 x \cdot 10^{-5}} \cdot 10$

\subsection{Experimental liquid absorbency rate of knitted spacer structures.}

For the experimental investigation of absorbency, different types of spacer knitted fabrics were produced on the 7 gauge Shima Seiki machine.

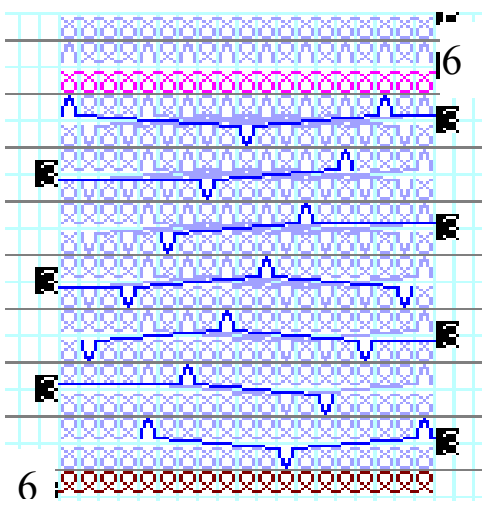

Spacer 5 ends of 167 dtex textured polyester. Indicated by number 3 on the stitch chart.

Outer yarn rear and front bed $=2$ ends of 167 dtex textured polyester. Indicated by the number 6 (rear) and number 2 (front) on the stitch chart.

Figure 5. Stitch diagram of spacer structures with polyester tuck spacer

For the purpose of achieving different porosity, capillary radii and angle of capillaries to the horizontal surface, spacer structures were produced by varying the number of spacer 
yarns in-between two single jersey courses and the number of spaces between two consecutive tucks. Figure 5 shows an example for one spacer fabric, which has 7 spacer yarns in-between two single jersey courses and 7 spaces in-between two consecutive tucks. Table 1 shows the fabric specification of different polyester spacer structures produced for our experimental and theoretical work. It also shows the capillary radius calculated using the model and the porosity based on the fabric sample weight, thickness and area, which is $50.26 \mathrm{~cm}^{2}$. The capillary radii given in Table 1 are calculated using the formulae (27).

Table 1. Fabric specification, capillary radius and porosity of some spacer samples

\begin{tabular}{|c|c|c|c|c|c|c|c|c|}
\hline Sample & Spaces & Thickness & Weight & Wales & Courses & Stitches & Cap.radii & Porosity \\
\hline & & $\mathrm{mm}$ & grams & per $\mathrm{cm}$ & per cm & Per $\mathrm{cm}^{2}$ & $\mu \mathrm{m}$ & \\
\hline $\mathrm{Sp}-21$ & 9 & 13.20 & 5.40 & 5.00 & 6.67 & 33.35 & 60.94 & 0.960 \\
\hline $\mathrm{Sp}-22$ & 10 & 13.35 & 5.35 & 4.60 & 6.67 & 30.68 & 55.63 & 0.954 \\
\hline $\mathrm{Sp}-23$ & 6 & 9.55 & 4.20 & 4.62 & 6.60 & 30.50 & 59.09 & 0.960 \\
\hline $\mathrm{Sp}-24$ & 7 & 10.20 & 4.69 & 4.81 & 6.29 & 30.30 & 56.69 & 0.957 \\
\hline $\mathrm{Sp}-25$ & 8 & 10.40 & 5.09 & 4.81 & 6.29 & 30.30 & 53.20 & 0.953 \\
\hline $\mathrm{Sp}-26$ & 9 & 10.15 & 5.15 & 4.63 & 5.93 & 27.50 & 51.64 & 0.952 \\
\hline $\mathrm{Sp}-27$ & 10 & 10.45 & 5.40 & 4.44 & 6.30 & 28.00 & 48.14 & 0.944 \\
\hline $\mathrm{Sp}-7$ & 12 & 10.30 & 6.60 & 5.00 & 5.60 & 28.00 & 48.86 & 0.931 \\
\hline $\mathrm{Sp}-8$ & 8 & 11.10 & 5.42 & 4.40 & 6.75 & 29.70 & 63.30 & 0.959 \\
\hline $\mathrm{Sp}-10$ & 6 & 9.10 & 4.52 & 4.40 & 7.00 & 30.20 & 58.12 & 0.957 \\
\hline $\mathrm{Sp}-11$ & 7 & 10.30 & 4.95 & 4.06 & 7.56 & 30.70 & 56.66 & 0.958 \\
\hline
\end{tabular}

A vertical absorbency test was carried out to measure the absorbency across the fabric for all eleven fabric samples after placing them horizontally on the porous plate of the absorbency tester and the results were recorded. Absorbency rate and total absorbency were compared with the fabric parameters and the theoretical absorbency.

\section{Results and discussion}

\subsection{Experimental results}


Figure 6 shows the experimental absorbency as grams of water per 100 grams of fabric whereas Figure 7 shows the absorbency in grams of water per $50 \mathrm{~cm}^{2}$. In the case of clothing material it is important to know the absorbency capacity per square unit of area as well as a percentage.

Figure 6 shows that the fabric samples with higher porosities have higher total absorbency. For example, sample Sp-21with porosity of 0.960 and sample Sp-8 with porosity of 0.959 have a higher total absorbency of about 1200\%. Similarly, fabric samples with lower porosities, Sp-7 with porosity of 0.931 and Sp-27 with porosity of 0.944 , have a lower total absorbency of about $900 \%$. The total absorbency of the other fabric samples also follows a similar sequence according to their porosity variation as given in Table 1.

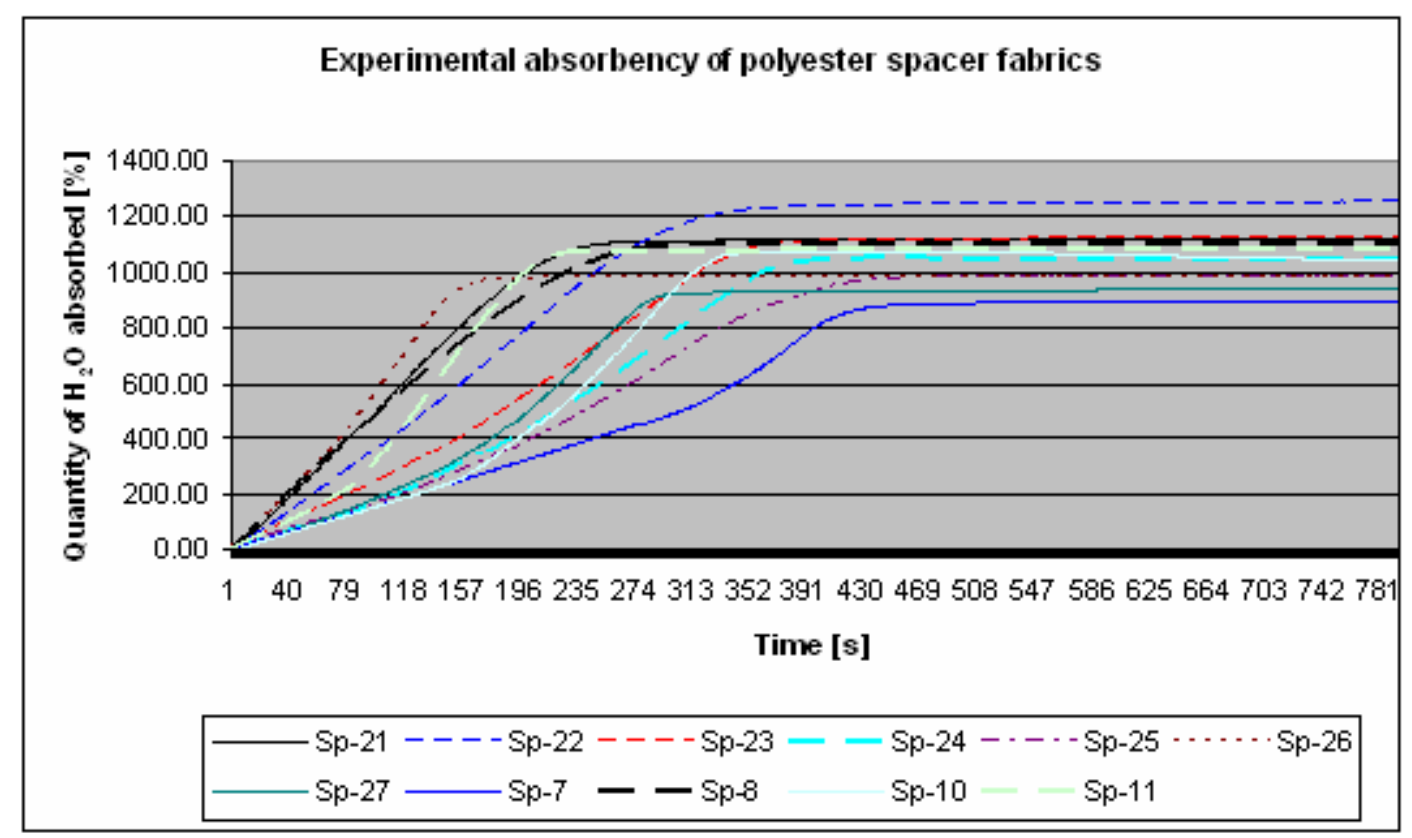

Figure 6 Experimental absorbency rates of spacer fabrics given in Table 1 as percentage

Figure 6 shows that the fabric samples Sp-21, Sp-22, Sp26 and Sp-8 have a higher and consistent absorbency rate from the beginning to saturation compared to the other 
structures in the table. This also shows that the absorbency rate between $400 \%$ absorbency and saturation is similar for most of the structures. However, the fabric samples having lower capillary radii and higher capillary angle $(\sin \varphi)$ to the horizon show lower absorbency rates.

Figure 7 shows that the absorbency per unit area of fabric samples follows a similar pattern in terms of percentage absorbency. However, the total absorbency per unit area seems to vary with the thickness of the fabric, the thicker the fabric the higher the total absorbency per unit area.

We observed that fabric sample Sp-7 had the lowest percentage absorbency but a higher absorbency per unit area compared to other samples. Sp-7 also has comparatively high fabric thickness.

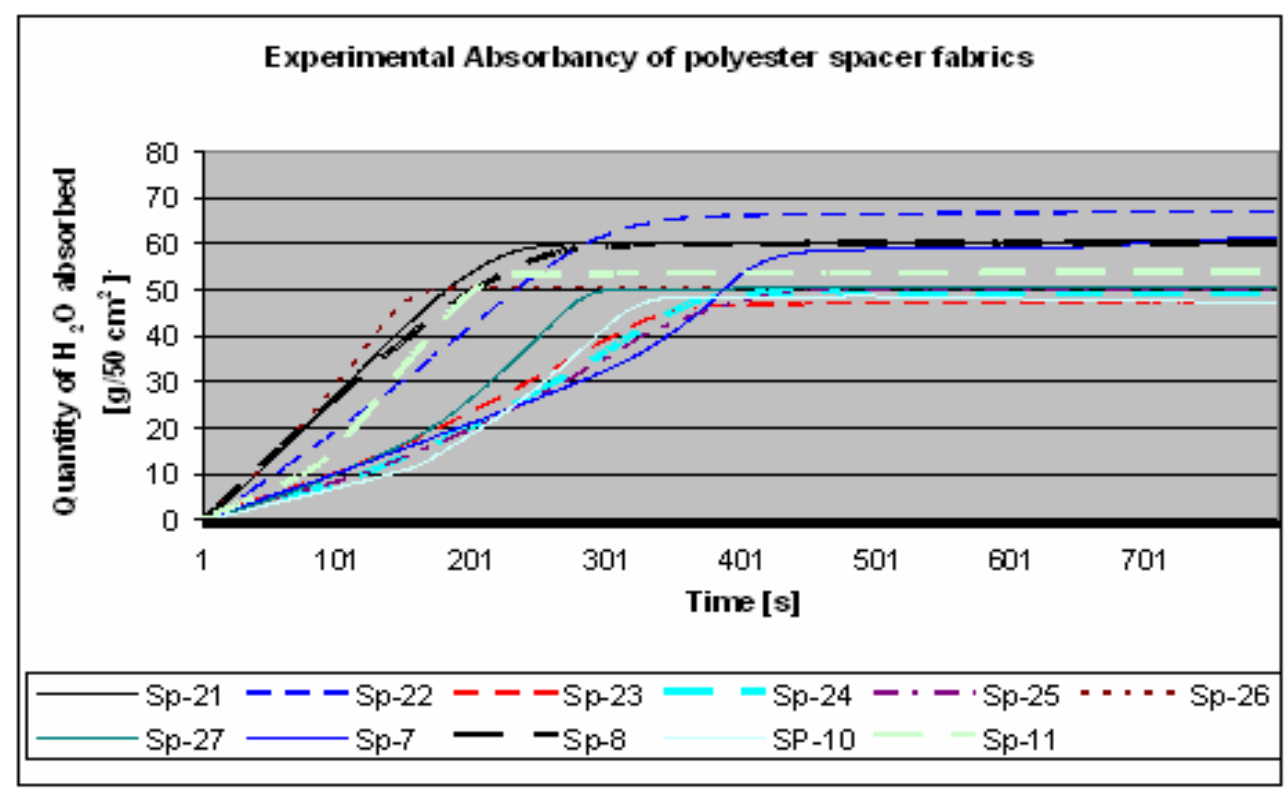

Figure 7 Absorbency of spacer fabrics given in Table 1 (grams per $50 \mathrm{~cm}^{2}$ )

\subsection{Theoretical liquid absorbency rate of knitted spacer structures}

First the capillary radii were calculated for all the fabric samples using the formula developed in the theoretical part and given in Table 1. 
Then the liquid transport along the capillaries was calculated and plotted against time using the liquid transport model, $\ell(\mathrm{t})=\mathrm{A} \cdot \operatorname{lambertw}\left(-\exp \left(-\left(\mathrm{K} \cdot \mathrm{B}^{2} \cdot \mathrm{t}+\mathrm{A}\right) / \mathrm{A}\right)+1\right) / \mathrm{B}$, developed in the theoretical study

The values for $A=\frac{2 \gamma}{r} \cos \theta, B=g \rho_{w} \frac{b}{\sqrt{b^{2}+z^{2}}}$ and $K=\frac{\left(r^{2}\right)}{8 \eta}$ were calculated as given in Table 3 using the constants given in Table 2 and fabric data from Table 1.

Table 2 Constant values for the properties of water

\begin{tabular}{|l|c|l|}
\hline Property & Symbol & Constant Value \\
\hline Density & $\rho_{\mathrm{w}}$ & $1.0 \mathrm{~g} / \mathrm{cm}^{3}$ \\
\hline Viscosity & $\eta$ & 0.1 dynessec $/ \mathrm{cm}^{2}$ \\
\hline Surface tension & $\gamma$ & $72.8 \mathrm{dynes} / \mathrm{cm}^{2}$ \\
\hline Gravity & $\mathrm{g}$ & $980 \mathrm{~cm} / \mathrm{sec}^{2}$ \\
\hline Contact angle (polyester) & $\cos \theta$ & $0.2588[80 \mathrm{a}]$ \\
\hline
\end{tabular}

The average contact angle is taken as $75^{\circ}$, which is the average of the minimum $\left(60^{\circ}\right)$ and maximum $\left(90^{\circ}\right)$ from the results of Lehocky, so the value of $\cos \theta$ for polyester is taken as 0.2588 .

Table 3 Specification of different fabric samples for absorbency tests

\begin{tabular}{|c|c|c|c|c|c|c|c|c|}
\hline 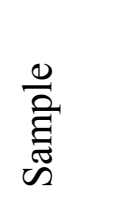 & 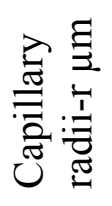 & 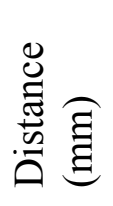 & $\stackrel{\ominus}{\ominus}$ & 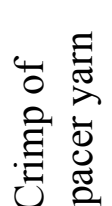 & $\varangle$ & $\infty$ & 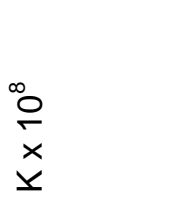 & 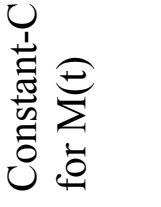 \\
\hline Sp-21 & 60.94 & 2.364 & 0.582 & 0.291 & 6183.021 & 569.990 & 4642.585 & 1389.280 \\
\hline Sp-22 & 55.63 & 2.674 & 0.518 & 0.271 & 6773.504 & 507.667 & 3868.428 & 1042.630 \\
\hline $\mathrm{Sp}-23$ & 59.09 & 1.612 & 0.592 & 0.225 & 6377.218 & 580.571 & 4364.141 & 1052.835 \\
\hline Sp-24 & 56.69 & 1.611 & 0.633 & 0.215 & 6647.364 & 620.354 & 4016.636 & 981.339 \\
\hline $\mathrm{Sp}-25$ & 53.20 & 1.624 & 0.640 & 0.201 & 7082.808 & 627.558 & 3537.940 & 872.719 \\
\hline Sp-26 & 51.64 & 1.646 & 0.617 & 0.171 & 7297.032 & 604.285 & 3333.257 & 841.152 \\
\hline Sp-27 & 48.14 & 1.708 & 0.612 & 0.187 & 7827.021 & 599.497 & 2897.132 & 692.395 \\
\hline $\mathrm{Sp}-7$ & 48.86 & 1.581 & 0.651 & 0.247 & 7712.856 & 638.286 & 2983.533 & 713.044 \\
\hline Sp-8 & 63.30 & 2.13 & 0.521 & 0.166 & 5952.828 & 510.649 & 5008.580 & 987.850 \\
\hline
\end{tabular}




\begin{tabular}{|l|l|l|l|l|l|l|l|r|}
\hline Sp-10 & 58.12 & 1.639 & 0.555 & 0.219 & 6483.884 & 543.982 & 4221.733 & 1008.966 \\
\hline Sp-11 & 56.66 & 2.008 & 0.513 & 0.134 & 6650.815 & 502.597 & 4012.468 & 869.597 \\
\hline
\end{tabular}
Where distance $=\sqrt{b^{2}+\frac{\text { spaces }^{2}}{w^{2}}}$ and spacer angle $\varphi, \sin \varphi=b / \sqrt{b^{2}+\frac{\text { spaces }^{2}}{w^{2}}}$

Table 3 shows the calculated capillary radii, the distance between two consecutive tucks on front and back bed, crimp of spacer yarn and the spacer angle.

The liquid take up rate is calculated using the following formula;

$$
\mathrm{M}(\mathrm{t})=\frac{170 \cdot \pi \cdot r^{2} \cdot \rho_{w} \cdot \ell(t) \cdot(1-\text { crimp })}{\sqrt{b^{2}+\frac{\text { spaces }^{2}}{w^{2}}} \cdot \operatorname{Tex} \cdot 10^{-5}} \cdot 100=\mathrm{C} \cdot \ell(\mathrm{t})
$$

The constant $\mathrm{C}$ is calculated using fabric parameters and the other constants. The count of the yarn used for the spacer was 83.5 tex $(5 \mathrm{x} 167 \mathrm{dtex})$ and the single jersey courses yarn in the front and back beds had the yarn count of 33.4 tex (2x167dtex). Table 3 gives the constant $\mathrm{C}$ for different fabric samples

The graphs in Figure 8 show the theoretical liquid take-up rate for different fabrics that we have already experimentally investigated. It also shows that the total absorbency varies from $800 \%$ to $1500 \%$ and that the absorbency rate of some structures is very high compared to other structures.

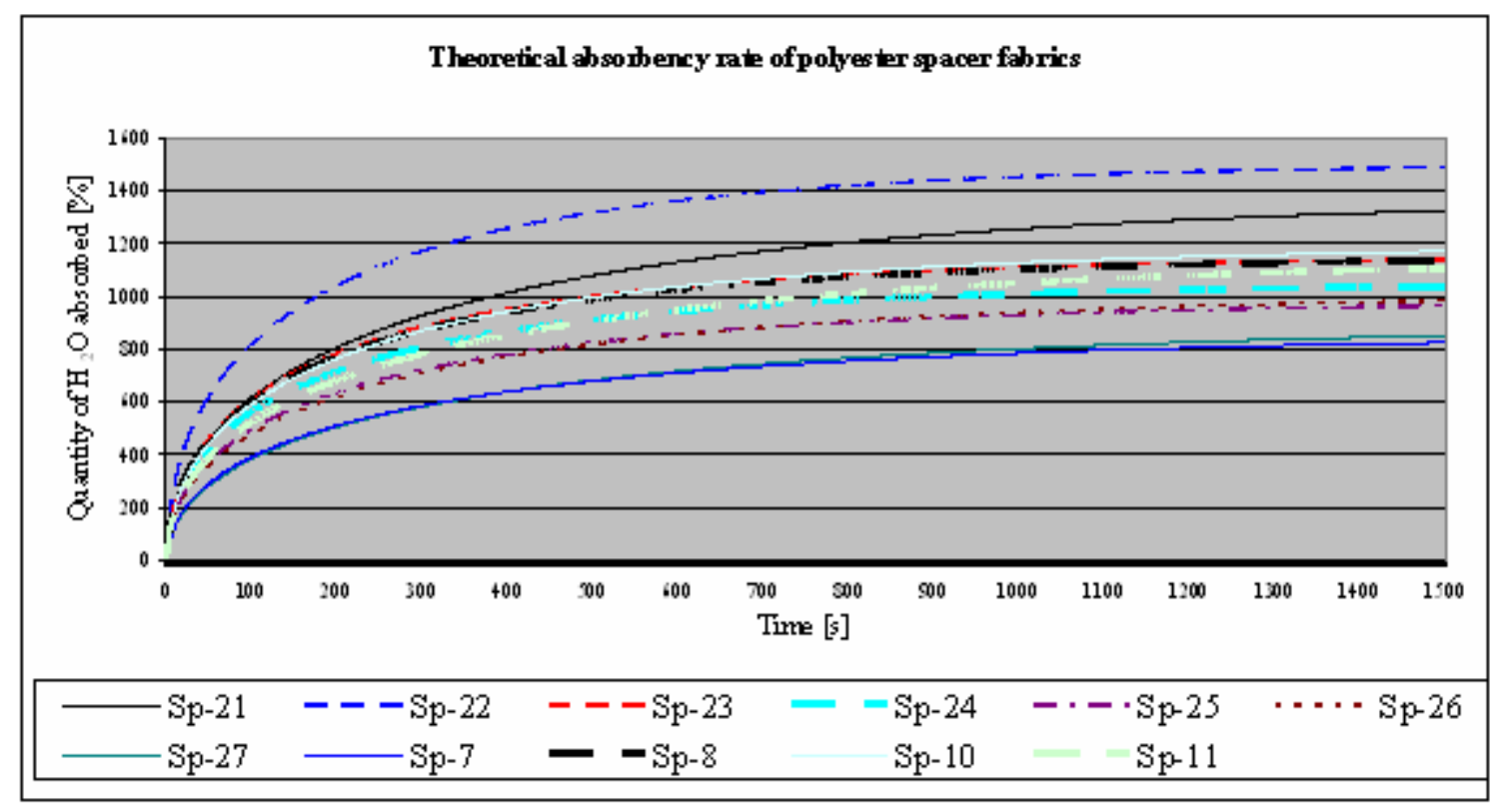


Figure 8 Theoretical absorbency rates of the spacer fabric samples

Figure 9 shows the comparison of theoretical and experimental absorbency of five selected spacer fabrics. $\mathrm{T}$ and $\mathrm{E}$ indicate the theoretical (continuous) and experimental (dotted) absorbency of selected fabric samples Sp-24, Sp-25, Sp-26, Sp-8 and Sp-11 respectively. It shows that the theoretical and experimental total absorbency are almost the same for the given structures.

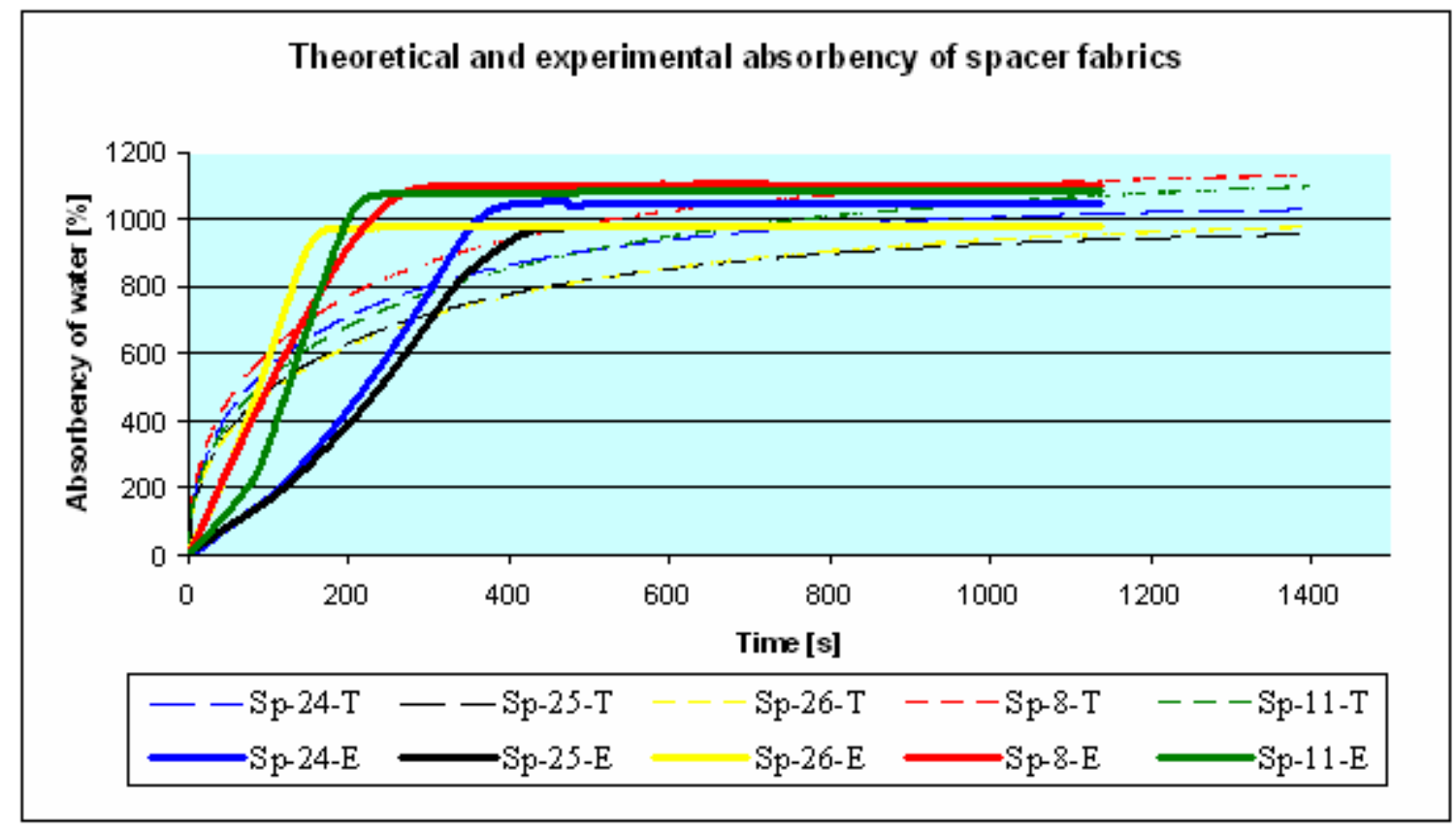

Figure 9 Theoretical and experimental curves of absorbency of selected spacer structures

When we compare the curves of experimental with the theoretical liquid take-up, the pattern is almost the same, although there are some variations at the beginning. These variations arise because the theoretical model assumes an average contact angle of $75^{\circ}$, independent of liquid rise. However, the research work carried out by Joos et. al shows that the contact angle is dynamic, having a value of $90^{\circ}$ at the beginning and going down to $65^{\circ}$ at saturation, which is known as the static contact angle [8]. Due to this fact the theoretical value of $2 \gamma \cos \theta$ in the equation (45) is higher than the actual value until the dynamic contact angle becomes $75^{\circ}\left(\cos 75^{\circ}=0.2588\right)$. As a result, the theoretical absorbency rate is higher than the experimental rate at the initial stage. Thereafter, when the dynamic contact angle goes further down, the theoretical value of $2 \gamma \operatorname{cose}$ becomes 
lower than the actual value. Therefore, after the dynamic contact angle reaches $75^{\circ}$, the theoretical absorbency rate is lower than the experimental values, until saturation is reached.

Figure 9 also shows that the time taken for saturation in both theoretical and experimental varies between 6 and 10 minutes for the given structures. The theoretical time taken for saturation is higher than the experimental time taken due to the same explanation given as for the absorbency rate after the dynamic contact angle of $75^{\circ}$.

\section{Conclusions}

The total water absorbed by a knitted structure depends on the porosity and other characteristics of the structure. Generally, knitted structures with high porosity absorb more water than those with low porosity.

Initially, the experimental absorbency rate is lower compared to the middle part of the curve for most of the structures; this may be due to the fabric surfaces of the spacer samples not being flat and not having full initial contact with the water surface, and taking time to reach full contact. However, in the case of heavy fabrics, the absorbency rate is more consistent. The absorbency capacity is not influenced by the contact surfaces. Theoretical absorbency values show that the structures with higher porosity absorb more water than structures with lower porosity. The water absorbency rate is lower in more compact structures which have finer capillaries and/or smaller pores than less compact structures.

The models developed to predict the absorbency in knitted spacer structure can be used directly to predict total absorbency in knitted spacer structures made with textured monofilament yarn.

The model can be also used to predict absorbency rate and the time taken for saturation. However, if dynamic contact angle is considered in the equations, the shape of the absorbency curve and saturation time can be predicted more accurately. 


\section{References}

1 Kissa,E., (1996). Wetting and Wicking. Textile research journal,. 66(10); p.660668

2 de Gennes, P.G., (1985). Wetting - Statics and Dynamics. Reviews of Modern Physics, 57(3): p.177-863.

3 Miller,B., (1985). in “Absorbency", by P.K. Chatterjee. Elsevier Science Publishers B.V., pp.121 - 124.

4 Yoo, Shunjung and Barker, Roger L., (2004). Moisture Management properties of Heat-Resistance workwear Fabrics. Textile Research Journal, 74(11), p.995 1000.

5 Washburn, E. W., (1921). "The dynamics of capillary flow". The Physical Review, 27(3), p. 273-283.

6 Laughlin, R. D. and Davies, J. E., (1961). Some Aspects of Capillary Absorption in Fibrous Textile Wicking. Textile Research Journal, 31(10), p. 904-910.

7 Fisher, L. R., (1979). An Experimental Study of the Washburn Equation for Liquid Flow in Very Fine Capillaries. Journal of Colloid and Interface Science, 69(3), p. 486-492.

8 Joos, P., Remoortere, P. V. and Bracke, M., (1990). The Kinetics of Wetting in a Capillary. Journal of Colloid and Interface Science, 136(1), p.189-197.

9 Jeje, A. A., (1979). Rates of Spontaneous Movement of Water in Capillary Tubes. Journal of Colloid and Interface Science, 69(3), p. 420-429.

10 Zhuang, Q., Harlock, S. C. and Brook, D. B., (2002). Longitudinal Wicking of Weft Knitted Fabrics Part II: Wicking Mechanism of Knitted Fabrics Used in Undergarments for Outdoor Activities. Journal of the Textile Institute: Fibre Science \& Textile Technology; 93(1), p 97-107. 
11 Hodgson, K. T. and Berg, J. C., (1988). The Effect of Surfactant on Wicking Flow in Fiber Networks. Journal of Colloid and Interface Science, 121(1), p.22-31.

12 Good, R.J and Lin, N.J., (1976). Rate of Penetration of a Fluid in to Pores Body 2, Verification of Generalisation Washburn Equation, for Organic-Liquid in Glass capillary. Journal of Colloid and Interface Science, 54(1), p.52-58.

13 Marmur, A. and Cohen, R.D., (1997). Characterisation of Porous Media by the Kinetics of Liquid Penetration: The vertical Capillary Model. Journal of Colloid and Interface Science, 189(2), p.299 - 304.

14 Nyoni, A.B. and Brook, D., (2006). Wicking mechanisms in yarns - the key to fabric wicking performance. Journal of the Textile Institute, 97(2), p119-128

15 Zohng, W., Ding, X and Tang, Z.L., (2001). Modelling and analysing liquid wetting in fibrous assemblies. Textile Research Journal, 71(9), p. 762- 766.

16 Saeed, U., (2006). The study of liquid transport behaviour of structures knitted with monofilament yarns. Unpublished masters thesis, School of Materials, The University of Manchester.

17 Fowler, J.L. and Hertel, K.L, (1940). Flow of a gas Through Porous Media. Journal of Applied Physics Physics, 11, p. 496- 502. 